\title{
CARACTERIZACIÓN DE LOS CROMOSOMAS POLITÉNICOS DE Simulium sp. QUEBRADA LA ALDANA RESERVA CEDRO ROSADO-QUINDIO
}

\author{
CHARACTERIZATION OF CHROMOSOMES polytene of Simulium sp. \\ QUEBRADA LA ALDANA PINK SEDAR RESERVE-QUINDIO
}

Mónica Vargas Montes ${ }^{1}$, Mateo Murillo ${ }^{1}$, Katherine Chacon ${ }^{1}$, Luisa Fernanda Arias ${ }^{1}$ y Nohra Rodríguez Castillo ${ }^{2}$

1. Estudiantes de Biología Universidad del Quindío.

2. MSc Docente Universidad del Quindío

Recibido: 20 de Septiembre de 2014

Aceptado: 15 de Octubre de 2014

*Correspondencia del autor.: nohrarodriguez@gmail.com

\section{RESUMEN}

Considerando la importancia de Simulium sp. como posible vector de Onchocerca volvulus, parásito causante de la oncocercosis, y teniendo en cuenta que el análisis de los cromosomas politénicos se ha convertido en un herramienta poderosa para la identificación y clasificación de la poblaciones vectoras o no vectoras; se realizó un estudio preliminar basado en la estandarización de la técnica de extracción de los cromosomas politénicos y la caracterización de estos para individuos del género Simulium sp. encontrados en la quebrada la Aldana de la Reserva Natural de Cedro Rosado, Quindío. Los resultados del estudio indicaron que la especie tiene una dotación n=3. La longitud relativa es 125 micras, de las cuales el $46 \%$ corresponde al cromosoma I, el 33\% al cromosoma II y el $21 \%$ al cromosoma III del complemento total. Los cromosomas I y II son submetacéntricos y el III metacéntrico. Se identifican los marcadores cromosómicos en la especie estudiada y un índice asimétrico=0,51.

Palabras claves: Simulium, cromosoma politénico, citogenética, citotaxonomía

\begin{abstract}
Considering the importance of Simulium sp. as a possible vector of Onchocerca volvulus, the parasite that causes oncocercosis, and considering that polytene chromosome analysis has become a powerful tool for identification and classification of vectors or vector populations, a preliminary study was carried out based on the standardization of the extraction technique polytene chromosomes and characterization of these for individuals Simulium sp. found in the Quebrada La Aldana, into Nature Reserve Cedro Rosado, Quindio. The results of the study indicated that the species has $\mathrm{n}=3$. The relative length is 125 microns, $46 \%$ corresponds to chromosome $\mathrm{I}, 33 \%$ to chromosome II and chromosome III $21 \%$ to total complement . Chromosomes I and II are submetacentric and metacentric III. Chromosomal markers are identified in the tested species and asymmetric index $=0.51$.
\end{abstract}

Keywords: Simulium, polytene chromosome, cytogenetic, cytotaxonomy 


\section{INTRODUCCIÓN}

En la familia Simulidae se encuentran alrededor de 1500 especies a nivel mundial, 300 están en el Neotrópico y 45 de éstas en Colombia, 33 del género $\mathrm{Si}$ mulium y 12 del género Gigantodax (1-3). En Colombia son limitados los estudios realizados en simúlidos y dada la posición geográfica del país, es posible que el número de especies sea mayor que el anotado (4). A nivel local se han realizado diferentes estudios donde se ha indicado la presencia de Simulium sp, como reporta Garcia-Alzate (5) en el rio Roble ubicado en el departamento del Quindío entre los municipios de Quimbaya y Montenegro, encontraron que Simulium $s p$ tiene una abundancia relativa en periodo de lluvias del 18,7\% y en el periodo seco 1,2\%. Del mismo modo Villareal y Sánchez (6) reportan a Simulium $s p$, en la quebrada la Aldana ubicada en el sendero Cedro Rosado de la universidad del Quindío.

Tidwell et al. (7) identificaron que Simulium exiguum, encontrado en el municipio López de Micay, es el vector de Onchocerca volvulus en Colombia; siendo esto de gran importancia biológica, ya que dicho parásito es causante de la enfermedad conocida como oncocercosis.

Con la introducción de la citogenética en el estudio del género Simulium, el análisis de los cromosomas politénicos de las larvas se han convertido en un herramienta poderosa para la identificación y clasificación de las poblaciones vectoras o no vectoras de este Díptero. (8).

Los cromosomas politénicos fueron observados por primera vez por E. G. Balbiani en 1881 (1) en núcleos interfásicos, que no completan la mitosis, por consiguiente los cromosomas se quedan estacionados en interfase, llegando a representar más de 1000 copias de una cromátide en un fenómeno llamado politenia. Cada cromosoma está constituido por un par de homólogos, íntimamente sinapsados, caracterizados por el patrón de bandas originado por la alineación de los diferentes cromómeros a todo lo largo del cromosoma. Es notable la constancia en la localización y distribución de los discos o bandas en los dos cromosomas homólogos. Es así muy fácil construir mapas topográficos de las bandas y de las interbandas y relacionarlos con los mapas genéticos. El patrón de bandas es específico para cada especie lo que permite observar cualquier anomalía de tipo estructural como deleciones, inversiones translocaciones y duplicaciones.

Niño (9) realizó estudios comparación de cromosomas politécnicos y ubicación marcadores cromosómicos en Simuliidae: Gigantodax basinflatus y Gigantodax sp. en la Quebrada la Vieja en el departamento de Cundinamarca-Colombia obteniendo como resultado un mapa citogenético por medio bandeo cromosómico extraído glándulas salivales de la mosca adulta, llegando a una comparación de las dos especies con una diferencia de distancias la ubicación de los marcadores.

La informacion obtenida a partir de herramientas citogeneticas tambien han contribuido con esta determinacion taxonomica, en el caso de Simulium, estos estudios se han basado en la presencia de cromosomas gigantes o cromosomas politénicos. Estos cromosomas se encuentran en diferentes órganos (glándulas salivales, intestino, recto, túbulos de Malpighi, ovarios, cuerpos grasos) de las larvas. En simulium estos cromosomas se localizan más fácilmente en las glándulas salivales de las larvas.

Teniendo en cuenta lo anterior, el propósito del actual estudio fue estandarizar el método de extracción de los cromosomas politénicos de las glándulas salivales de la larva de Simulium sp. encontrada en la quebrada la Aldana de la Universidad del Quindío; y a su vez realizar la caracterización de los cromosomas extraídos.

\section{METODOLOGÍA \\ Área de estudio}

Teniendo en cuenta estudios previos que reportan la presencia de Simulium sp. se escogió la quebrada la Aldana en la reserva Natural Cedro Rosado localizada en el campus de la Universidad del Quindío como lugar de muestreo.

La Reserva se encuentra al noroeste de la ciudad de Armenia en la vertiente occidental de la cordillera Central de los Andes colombianos $\left(4^{\circ} 32^{\prime} 40^{\prime} \mathrm{N}\right.$; $\left.75^{\circ} 46^{\prime} 13^{\prime} \mathrm{W}\right)$. Tiene una extensión aproximada de 15 ha. Se encuentra a una altura entre los 1490 y 1530 msnm., el clima presenta una distribución bimodal de lluvias, con dos periodos de máxima precipitación en los meses de marzo-mayo y septiembre-noviembre, y dos periodos de menores precipitaciones entre diciembre-febrero y junio-agosto. El promedio anual 
de precipitación es de $2436 \mathrm{~mm}$, la temperatura es de $19^{\circ} \mathrm{C}$, con una humedad relativa entre 65 y $75 \%$, por lo cual la zona de vida es de bosque muy húmedo premontano (bhm-P) (10).

\section{Recolección del material biológico}

Se recolectaron larvas de Simulium $s p$ en la quebrada mencionada anteriormente, por el método de colecta directa, teniendo en cuenta que se localizan donde la corriente del río y la caída del agua son muy fuertes y están adheridas sobre piedras, hojas, troncos o plásticos a poca profundidad. Las larvas fueron fijadas en Carnoy y otras en etanol al 95\% y fueron transportadas en frío a $4{ }^{\circ} \mathrm{C}$ hasta el laboratorio.

\section{Disección de las larvas de Simulium sp.}

Para la disección de las glándulas salivares, en una lámina con unas gotas de fijador diluido, se colocó una larva de último estadio (con su histoblasto maduro) bajo estereoscopio y utilizando dos agujas de disección de punta fina la larva se perforó el integumento por la parte ventral, con una escisión desde el sexto hasta el noveno segmento abdominal (2/3 partes ostero-ventral del abdomen). Una vez abierta la pared corporal, se localizaron fácilmente las glándulas salivares a lado y lado del intestino, reconocidas como dos bolsas alargas, recubiertas o rodeadas de cuerpos grasos, que se retirarse posteriormente. Se fijaron las glándulas con unas gotas de Carnoy por dos o tres minutos. Se retiraron las glándulas, a una lámina limpia en la cual se adicionaron unas gotas de ácido acético al 50\%, para fragmentar el tejido. Posteriormente se agregaron unas 3-4 gotas de orceína lactoacética para tincionar las células por 5 minutos. Se sobrepuso la laminilla y se efectuó el esparcimiento del tejido por un simple movimiento de rotación del dedo índice sobre la laminilla, entre dos papeles absorbentes (toallas de papel)(11).

Una vez logrados los montajes se realizaron los estudios citogenéticos que para este caso consistieron en la evaluación del tamaño cromosómico con ayuda del software IMAGE J, sobre imágenes logradas con la cámara fotográfica CANON REBEL T3 adaptada al microscopio marca ZEISS AXIO SCOPE. Sobre los registros fotográficos se identificaron las bandas e interbandas cromosómicas para determinar los segmentos a lo largo del cromosoma. Adicionalmente se identificaron marcadores cromosómicos teniendo en cuenta la metodología descrita por Moreno (12-13), Muñoz (14), Henry et al. (15), y Chaliow et al. (16) y
Mustapha et al. (17).

Los cromosomas fueron clasificados teniendo en cuenta la nomenclatura propuesta por Levan (18) y se determinó la Asimetría Intracromosómica de acuerdo al método establecido por Romero \& Zarco (19) mediante la fórmula:

$$
\mathrm{A} 1=1-\sum \frac{b 1 / B 1}{n}
$$

$$
\text { b1: Longitud promedio del brazo corto }
$$

Donde: B1: Longitud promedio del brazo largo n: Número de pares de cromosomas homólogos

\section{RESULTADOS}

Se recolecto aproximadamente 500 larvas de Simulium sp. Se diseccionaron adecuadamente 100 de las 500 larvas de Simulium sp. recolectadas y los preparados permitieron la identificación de una dotación cromosómica $n=3$. El cromosoma I (Figura 1), presenta una longitud relativa de 57,39 micras, que se subdividen en 70 segmentos (Tabla1).

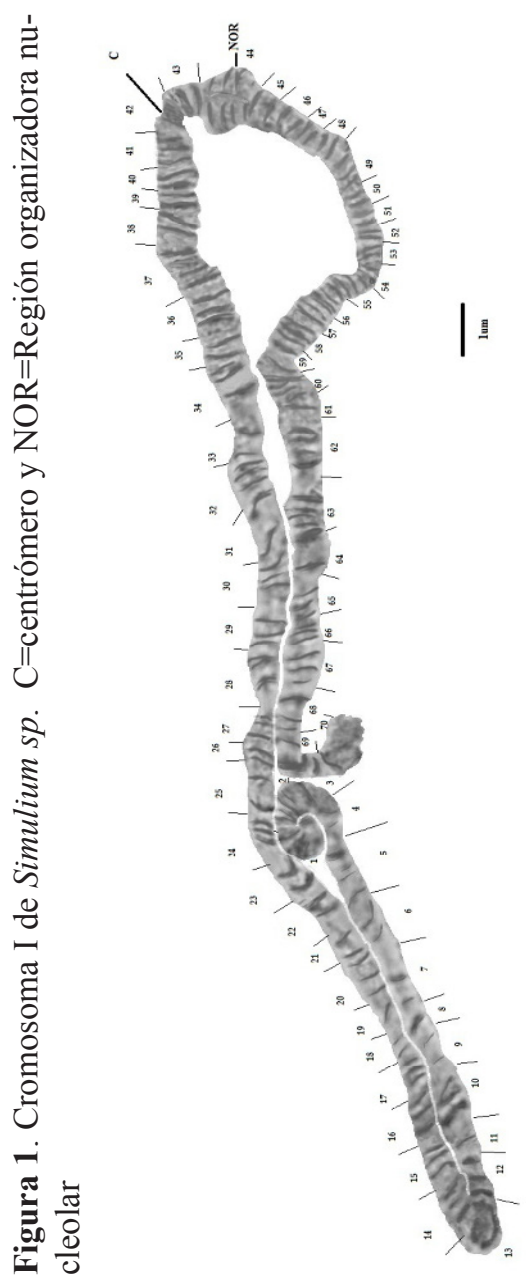


Tabla 1. Longitudes relativas del cromosoma I

\begin{tabular}{|c|c|c|c|c|}
\hline Segmento & PERIM & ANGLE & CIRC & LENGTH(UM) \\
\hline$\overline{1}$ & 62,8 & 162.4 & 0.2 & 0,93202731 \\
\hline 2 & 86,6 & 9.2 & 0.1 & 1,28524785 \\
\hline 3 & 72 & -89.2 & 0.2 & 1,06856634 \\
\hline 4 & 58,6 & -162.2 & 0.2 & 0,86969427 \\
\hline 5 & 91 & -168.6 & 0.1 & 1,35054912 \\
\hline 6 & 74,8 & -168.5 & 0.2 & 1,1101217 \\
\hline 7 & 71,2 & -167.1 & 0.2 & 1,05669338 \\
\hline 8 & 36,2 & -173.7 & 0.4 & 0,53725141 \\
\hline 9 & 57 & -169.9 & 0.2 & 0,84594835 \\
\hline 10 & 75,8 & -169.3 & 0.2 & 1,1249629 \\
\hline 11 & 53,6 & 174.6 & 0.2 & 0,79548828 \\
\hline 12 & 61,5 & -176.3 & 0.2 & 0,91273375 \\
\hline 13 & 126,3 & 135.3 & 0.1 & 1,87444346 \\
\hline 14 & 72,1 & 33.7 & 0.2 & 1,07005046 \\
\hline 15 & 63,2 & 18.4 & 0.2 & 0,93796379 \\
\hline 16 & 58,6 & 11.9 & 0.2 & 0,86969427 \\
\hline 17 & 51,9 & 25.1 & 0.2 & 0,77025824 \\
\hline 18 & 45,4 & 24.9 & 0.3 & 0,67379044 \\
\hline 19 & 42,6 & 19.3 & 0.3 & 0,63223508 \\
\hline 20 & 60,3 & 34.8 & 0.2 & 0,89492431 \\
\hline 21 & 41,2 & 25.9 & 0.3 & 0,61145741 \\
\hline 22 & 61,5 & 41.0 & 0.2 & 0,91273375 \\
\hline 23 & 58,5 & 47.1 & 0.2 & 0,86821015 \\
\hline 24 & 71,3 & 8.1 & 0.2 & 1,0581775 \\
\hline 25 & 69,4 & 3.3 & 0.2 & 1,02997922 \\
\hline 26 & 22,7 & 0 & 0.6 & 0,33689522 \\
\hline 27 & 50,5 & -12.4 & 0.3 & 0,74948056 \\
\hline 28 & 78,3 & 14.0 & 0.2 & 1,16206589 \\
\hline 29 & 60,9 & -10.4 & 0.2 & 0,90382903 \\
\hline 30 & 61,3 & 0 & 0.2 & 0,90976551 \\
\hline 31 & 59,6 & 10.6 & 0.2 & 0,88453547 \\
\hline 32 & 74,8 & 22.1 & 0.2 & 1,1101217 \\
\hline 33 & 55,1 & -6.2 & 0.2 & 0,81775007 \\
\hline 34 & 85,1 & 32.6 & 0.1 & 1,26298605 \\
\hline 35 & 40,1 & 14.4 & 0.3 & 0,59513209 \\
\hline 36 & 59,2 & 22.7 & 0.2 & 0,87859899 \\
\hline 37 & 84 & 35.8 & 0.2 & 1,24666073 \\
\hline 38 & 42,7 & 0 & 0.3 & 0,6337192 \\
\hline 39 & 21,4 & -2.7 & 0.6 & 0,31760166 \\
\hline 40 & 28,8 & 12.1 & 0.5 & 0,42742654 \\
\hline 41 & 48,7 & -9.5 & 0.3 & 0,7227664 \\
\hline 42 & 68,6 & -24.0 & 0.2 & 1,01810626 \\
\hline 43 & 54,5 & -68.6 & 0.2 & 0,80884535 \\
\hline 44 & 88,5 & -96.5 & 0.1 & 1,31344613 \\
\hline 45 & 34,2 & -110.6 & 0.4 & 0,50756901 \\
\hline 46 & 42,2 & -108.0 & 0.3 & 0,6262986 \\
\hline 47 & 25,9 & -125.5 & 0.5 & 0,38438706 \\
\hline 48 & 35 & -106.9 & 0.4 & 0,51944197 \\
\hline 49 & 69,8 & -153.8 & 0.2 & 1,0359157 \\
\hline 50 & 37,9 & -161.6 & 0.3 & 0,56248145 \\
\hline 51 & 30,5 & -156.8 & 0.4 & 0,45265657 \\
\hline 52 & 32,2 & -172.9 & 0.4 & 0,47788661 \\
\hline 53 & 30,7 & -178.2 & 0.4 & 0,45562481 \\
\hline 54 & 37,6 & 156.8 & 0.3 & 0,55802909 \\
\hline 55 & 28,1 & 120.3 & 0.5 & 0,4170377 \\
\hline 56 & 24,5 & 151.4 & 0.5 & 0,36360938 \\
\hline 57 & 24 & 125.0 & 0.5 & 0,35618878 \\
\hline 58 & 34 & 137.4 & 0.4 & 0,50460077 \\
\hline 59 & 40,8 & 168.7 & 0.3 & 0,60552093 \\
\hline 60 & 27,4 & -132.0 & 0.5 & 0,40664886 \\
\hline 61 & 45,5 & 174.9 & 0.3 & 0,67527456 \\
\hline 62 & 83,2 & 173.1 & 0.2 & 1,23478777 \\
\hline 63 & 70,1 & 170.9 & 0.2 & 1,04036806 \\
\hline 64 & 73,3 & 178.4 & 0.2 & 1,0878599 \\
\hline 65 & 41,8 & 157.7 & 0.3 & 0,62036213 \\
\hline 66 & 34,9 & -173.5 & 0.4 & 0,51795785 \\
\hline 67 & 67,6 & 164.5 & 0.2 & 1,00326506 \\
\hline 68 & 59,9 & 159.4 & 0.2 & 0,88898783 \\
\hline 69 & 63,2 & -137.5 & 0.2 & 0,93796379 \\
\hline 70 & & $65-25.4$ & 0.2 & 0,96467795 \\
\hline
\end{tabular}

El cromosoma II (Figura 2), presenta una longitud de 41,20 micras, que se subdividen en 46 segmentos (Tabla2).

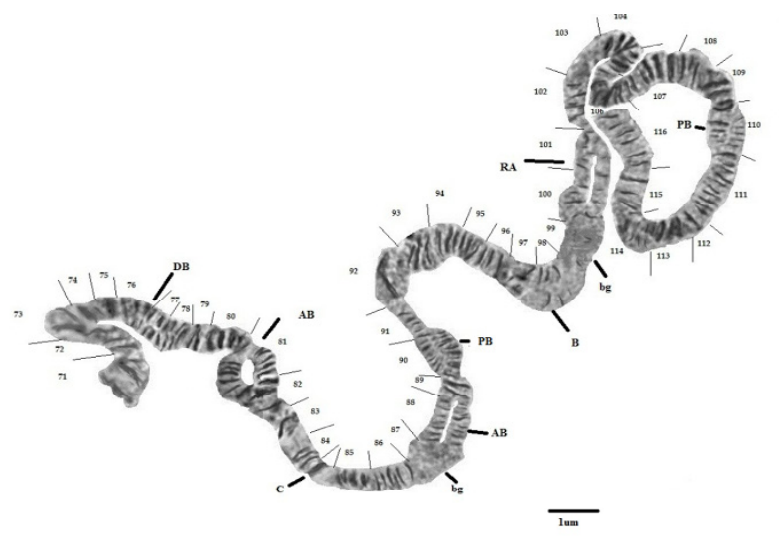

Figura 2. Cromosoma II. $\mathrm{DB}=$ doble bucle, $\mathrm{AB}=$ anillo de Balbiani, $\mathrm{C}=$ centrómero, $\mathrm{bg}=$ banda gris, $\mathrm{PB}=$ anillo parabalbiani, $\mathrm{RA}=$ Región Asináptica

Tabla 2. Longitudes relativas en el cromosoma II

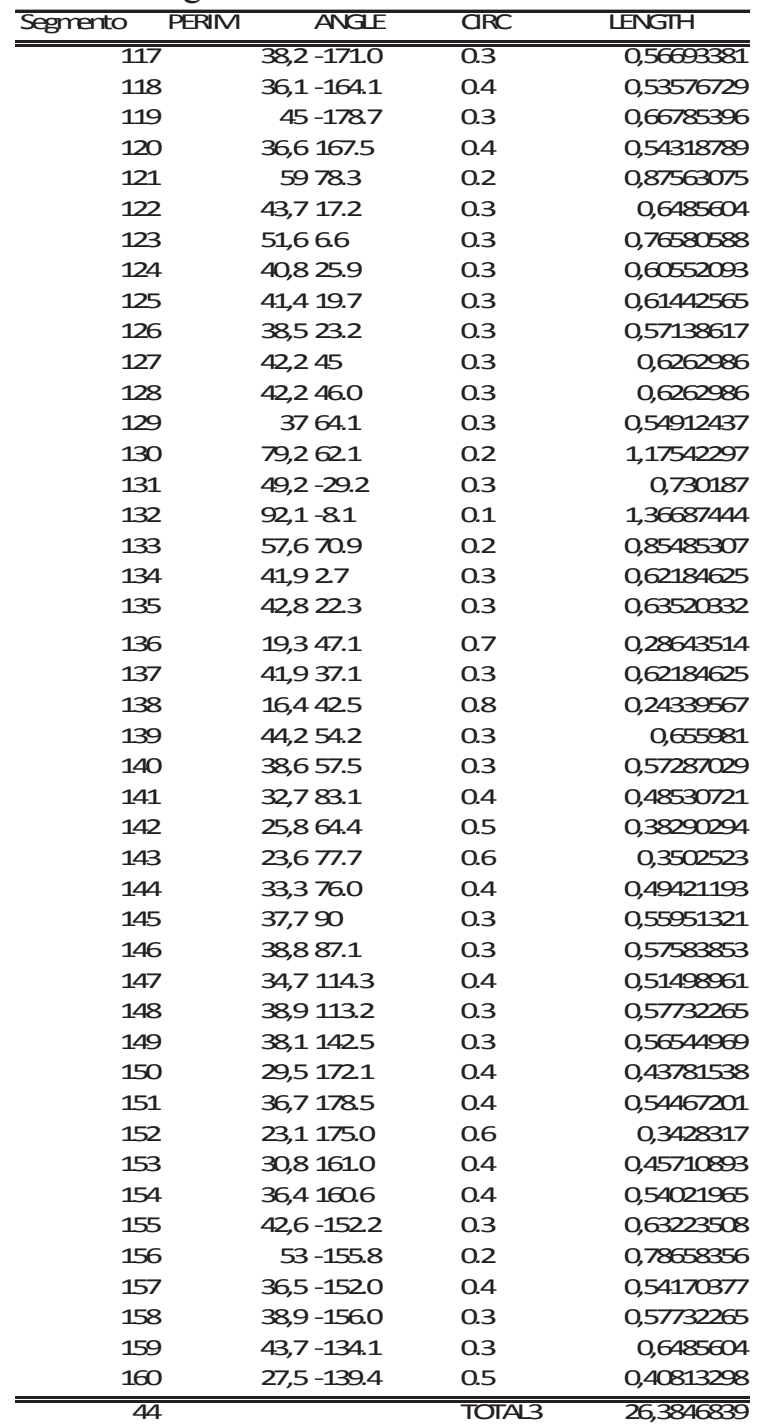


El cromosoma III (Figura 3), presenta una longitud de 26,38 micras, que se subdividen en 44 segmentos (Tabla 3).

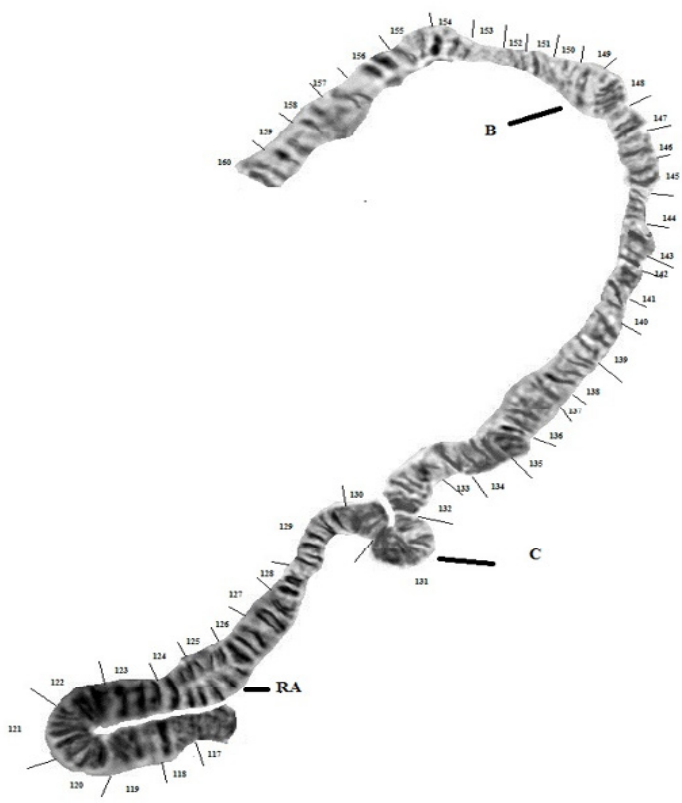

\begin{tabular}{|c|c|c|c|}
\hline Segrento PERIM & ANGF & aRC & LENGTH \\
\hline 1177 & $38,2-1710$ & 20.3 & 0,56693381 \\
\hline 118 & $36,1-164.1$ & 0.4 & $0,53576 / 29$ \\
\hline 119 & $45-1787$ & 0.3 & $0,66 / 85396$ \\
\hline 120 & $36,616.5$ & 0.4 & 0,54318789 \\
\hline 121 & 59783 & 0.2 & $0,8 / 563075$ \\
\hline 122 & $43,717.2$ & 0.3 & 0,6485604 \\
\hline 123 & 51,666 & 0.3 & 0,76580588 \\
\hline 124 & $40,825.9$ & 0.3 & 0,60552093 \\
\hline 125 & 41,4 19.7 & 0.3 & 0,61442565 \\
\hline 126 & $38,523.2$ & 0.3 & 0,57138617 \\
\hline 127 & 42,245 & 0.3 & 0,6262986 \\
\hline 128 & 42,2460 & 0.3 & 0,6262986 \\
\hline 129 & 3764.1 & 0.3 & 0,54912437 \\
\hline 130 & 79,2621 & 0.2 & 1,17542297 \\
\hline 131 & $49,2-29.2$ & 0.3 & 0,730187 \\
\hline 132 & $92,1-81$ & 0.1 & 1,36687444 \\
\hline 133 & $57,670.9$ & 0.2 & 0,85485307 \\
\hline 134 & 41,927 & 0.3 & 0,62184625 \\
\hline 135 & 42,8223 & 0.3 & 0,63520332 \\
\hline 136 & $19,347.1$ & 0.7 & 0,28643514 \\
\hline 137 & $41,937.1$ & 0.3 & 0,6184625 \\
\hline 138 & 16,4425 & 0.8 & 0,24339567 \\
\hline 139 & $44,254.2$ & 0.3 & 0,655981 \\
\hline 140 & $38,657.5$ & 0.3 & $0,5 / 287029$ \\
\hline 141 & $32,783.1$ & 0.4 & 0,48530721 \\
\hline 142 & $25,864.4$ & 0.5 & 0,38290294 \\
\hline 143 & $23,677.7$ & 0.6 & 0,3502523 \\
\hline 144 & 33,3760 & 0.4 & 0,49421198 \\
\hline 145 & 37,790 & 0.3 & 0,55951321 \\
\hline 146 & $38,887.1$ & 0.3 & $0,5 / 583853$ \\
\hline 147 & $34,7114.3$ & 0.4 & 0,51498961 \\
\hline 148 & $38,9113.2$ & 0.3 & $0,5 / 732265$ \\
\hline 149 & 38,11425 & 0.3 & 0,56544969 \\
\hline 150 & 29,51721 & 0.4 & 0,43781538 \\
\hline 151 & 36,71785 & 0.4 & $0,5446 / 201$ \\
\hline 152 & $23,1175.0$ & 0.6 & 0,3428317 \\
\hline 153 & 30,81610 & 0.4 & 0,45710893 \\
\hline 154 & $36,4160.6$ & 0.4 & 0,54021965 \\
\hline 155 & $42,6-1522$ & 0.3 & 0,63223508 \\
\hline 156 & $53-155.8$ & 0.2 & 0,78658356 \\
\hline 157 & $36,5-1520$ & 0.4 & 0,54170877 \\
\hline 158 & $38,9-156.0$ & 0.3 & $0,5 / 732265$ \\
\hline 159 & $43,7-134.1$ & 0.3 & 0,6485604 \\
\hline 160 & $27,5-139.4$ & 0.5 & 0,40813298 \\
\hline 44 & & $\overline{\text { TOTA }}$ & 26,3846839 \\
\hline
\end{tabular}

Figura 3. Cromosoma III. RA=Región Asináptica, $\mathrm{C}=$ centrómero, $\mathrm{B}=\mathrm{Blister}$

De acuerdo a las normas de Levan (18), los cromosomas 1 y 2 son submetacéntricos con proporciones de brazos de 2,09 y 2,78 respectivamente y el cromosoma 3 es metacéntrico con una relación de brazos de 1,61 (Tabla 4).

Tabla 4. Longitudes relativas en los cromosomas de Simulium sp.

\begin{tabular}{|c|c|c|c|}
\hline & cromosoma I & cromosoma II & cromosoma III \\
\hline$\overline{\text { longitudp }}$ & 18,51884832 & 10,87859899 & 10,10240427 \\
\hline longitudq & 38,87948946 & 30,33095874 & 16,28227961 \\
\hline \multicolumn{4}{|l|}{ Longitud } \\
\hline relativa & 57,39833779 & 41,20955773 & 26,38468388 \\
\hline \multicolumn{4}{|l|}{ Proporcion de } \\
\hline brazos q/p & 2,099455041 & 2,788130969 & 1,611723226 \\
\hline \multicolumn{4}{|l|}{ IC:Indice } \\
\hline \multicolumn{4}{|l|}{ Centromerico $=$} \\
\hline $100(p / p+q)]$ & 67,73626374 & 26,39824252 & 38,28889639 \\
\hline Clasificación & submetacentrico & submetacentrico & metacentrico \\
\hline
\end{tabular}

$\overline{\text { Metacéntrico } 1 \text { a } 1.7 \text {; submetacéntrico, } 1.7 \text { y } 3 \text {; subtelocéntricos entre } 3 \text { y }}$ 7 ; y acrocéntricos $>7$

La longitud relativa de los cromosomas politénicos en la especie estudiada comprende aproximadamente 125 micras, de las cuales el $46 \%$ corresponde al cromosoma I, el $33 \%$ al cromosoma II y el $21 \%$ al cromosoma III del complemento total.

El cromosoma I, presenta un brazo corto con una longitud relativa de 18,5 micras y un brazo largo de 38,8micras. En este cromosoma se identificaron dos marcadores cromosómicos, el centrómero en el segmento $42 / 43$ y la región organizadora nucleolar en el brazo corto, en el segmento 44 .

El cromosoma II, presenta un brazo corto con una longitud relativa de 10,8 micras y un brazo largo de 30,3micras. En este cromosoma se identificaron 9 marcadores cromosómicos, el centrómero en el segmento $84 / 85$, en el brazo corto un doble bucle en el segmento 76/77, y el anillo de Balbiani, en los segmentos 81/82. Y en el brazo largo dos anillos de Parabalbiani en los segmentos 90 y 110 , dos regiones de banda gris localizadas en los segmentos 87 y 99, un Blister en el segmento 97/98 y una región asináptica en el segmento 100/101.

El cromosoma III, presenta un brazo corto con una 
longitud relativa de 10,1 micras y un brazo largo de 16,3 micras. En este cromosoma se identificaron 3 marcadores cromosómicos, el centrómero en el segmento 131, en el brazo corto una región asináptica en el segmento $124 / 125$ y en el brazo largo un blíster en el segmento 148/149.

\section{Discusión}

La buena cantidad de larvas recolectadas (500 larvas de Simulium sp.), indica que las características físicoquímicas de la quebrada "La Aldana" son buenas desde el punto de vista del nivel trófico, ya que esta especie es indicadora de la calidad de las aguas y se localiza en sistemas con buena aireación, es importante tener en cuenta que a la quebrada son arrojados los desechos de la universidad sin previo tratamiento de estos residuos.

En la estandarización de la técnica citogenética es clave el proceso de fijación, el cual se realizo con carnoy 3:1 a bajas temperaturas, condiciones bajo las cuales el proceso de deshidratación celular por el etanol es lento y el reemplazamiento de agua por acido acético permite mantener las estructuras celulares en buen estado.

Una vez obtenidas buenas placas (láminas) y registros fotográficos, se evaluaron las características citogenéticas, teniendo en cuenta lo reportado en la literatura, encontramos que los segmentos denominados como duplicaciones, son regiones de bandas e interbandas que tienen relación con las zonas de genes activos (bandas claras) y no activos y/o zonas de regulación de la expresión de genes (bandas oscuras). Encontrándose que no hay una relación uno a uno de bandas e interbandas. Según Munoz (14), los cromosomas son divididos hasta 100 segmentos, sin embargo al analizar las bandas se identificaron en este estudio $160 \mathrm{seg}$ mentos de los cuales, el autor, indica que se dividen en segmentos más o menos uniformes por cromosoma 42 (cromosoma I), 30(cromosoma II) y 28 (cromosoma III), valores que difieren significativamente $(\mathrm{p}<0.05)$ de los observados en el presente estudio 70(cromosoma I), 45(cromosoma II) y 43(cromosoma III). Lo anterior indica variabilidad en las especies presentes en la Quebrada La Aldana, por lo cual es importante ampliar las zonas de muestreo.

Los marcadores cromosómicos son específicos dentro de las especies, las regiones de síntesis de ARN como los NOR, y los Puffs que son regiones engrosadas que se denominan anillos de Parabalbiani, con la cromatina poco extendida y los Anillos de Balbiani que presentan la cromatina muy distendida, se localizan a lo largo de los cromosomas, generalmente asociados, los dos últimos, al cromosoma II, en nuestra especie encontramos un NOR (cromosoma I), 2 Anillos de Balbiani, y 2 Anillos de Parabalbiani (cromosoma II).

Pelling (20), estableció que estos puffs, son sitios de transcripción, y su tamaño depende de la actividad de las bandas o interbandas que lo forman. En nuestro estudio se observo que el anillo de Balbini del segmento $81 / 82$ es más pequeño (1,63um) que el del segmento $88 / 89$ (1,95 um), indicando esto la transcripción diferencial de tales segmentos.

Marcadores cromosómicos similares fueron localizados por Henry et al. (15), Chaliow et al. (16) y Mustapha et al. (17), excepto por que en el cromosoma II se encontraron dos anillos de Balbiani y dos zonas de bandeo gris. En cuanto a la clasificación, de los cromosomas, el autor establece que los cromosomas I y II son metacéntricos y el III submetacéntrico, en el presente estudio, los resultados fueron diferentes, los cromosomas I y II son submetacéntricos y el cromosoma III metacéntrico. Lo anterior se puede explicar por el grado de condensación de los cromosomas al momento de la fijación, y por otro lado es una posible evidencia de que estos caracteres morfológicos de los cromosomas determinan que esta especie es diferente de la que trabajaron (15-17), siendo un carácter taxonómico que es necesario validar mediante la comparación con otras poblaciones distribuidas en una zona geográfica mayor.

Las regiones de asinapsamientos varían de un individuo a otro, estas determinan el grado de heterocigocidad en las poblaciones, convirtiéndose estas regiones en las zonas que evidencian la hibridación, en este estudio se logro establecer que los diversos individuos evaluados presentaban amplias zonas de asinapsamiento, lo cual determina el alto flujo génico en la especie y su potencial dispersamiento como especie vectora de múltiples parásitos de tipo filárico(nematodos) que afectan animales domésticos $y$ al hombre.

El índice de asimetría Intracromosómica dio como resultado cariotipos asimétricos con un valor de A1 $=0,51$. De acuerdo al resultado de las asimetrías cromosómicas se corrobora lo planteado por Romero- 
Zarco (19) que cuando los índices se acercan a cero los cromosomas son en su mayoría metacéntricos y los cariotipos son simétricos. Lo cual es corroborado en la clasificación metacéntrica y submetacéntrica al usar la relación de brazos propuesta por Levan (18).

Por lo tanto, se concluye que la metodología evaluada permite la caracterización de los cromosomas en la especie y se recomienda determinar la especie a través de un especialista en dípteros, y adicionalmente evaluar otras poblaciones de Similium $s p$. para determinar la utilidad de la citogenética en su esclarecimiento taxonómico y a determinación de las especies vectoras de parásitos filáricos.

\section{BIBLIOGRAFIA}

1. Muñoz P., Bueno M.L. y Moncada L.I. (1982). Simuliidae (insecta: díptera) de Colombia, especies de simulidos registradas en Colombia. Scientia I. 141-146

2. Crosskey, R.W. (1987). Black fly species of the word. Part VIII. 32 An annotated checklist of the world black flies(Diptera: Simuliidae)425-520. In Black flies: Ecology, Population management and annotated world list. Eds. K.C.Kim and R.W. Merrit. The Pensylvania State University. Press, University Park. 528p.

3. Wigodzinsky P. y Coscarón S. (1989).Description of three unusual species of the black fly subgenus Simulium (Ectemnaspis) from the Andes of Colombia (Diptera: Simuliidae). In: American Museum of natural History, Nov 2736: 1-13

4. Campos J y Muñoz P. (1990) Los cromosomas politénicos de Simulium furcillatum (Diptera: Simulidae) Chisaca, Cundinarma, Colombia. Rev. Acad. Colomb. Cienc. 17 (67): 715:723.

5. Garcia C A, Román C, Taphorn D y Gonzalez M. (2010) Physicochemical and biological characterization of the Roble river, Upper Cauca, western Colombia. Rev. Mus. Argentino Cienc. Nat., n.s. 12(1): 5-16, Buenos aires, ISSN 1514-5158.

6. Villareal A y Sanchez J A, (2009). Macroinvertebrados acuaticos como un sistema de evaluación de la calidad de aguas de la quebrada Cedro Rosado. On line consultada el 02/12/11 disponible en: http://issuu.com/alejovillarreal/docs/ macroinvertebrados como bioindicadores.

7. Tidwell M A, Tidwell M, Muñoz P y Corredor A. (1980). Simulium exiguum, the vector of Onchocerca volvulus on the Río Micay, Colombia. Am J Trop Med Hyg 29(3):377- 381.

8. Dunbar, R.W. (1966). Four sibling species includes in Simulium damnosum Theobald (Diptera: Simuliidae) from Uganda. Nature, 209:597-9

9. Niño E. A. (2003) Caracterización, comparación de cromosomas politécnicos y ubicación de marcadores cromosomáticos en Gigantodax basinflatus Y Gigantodax sp. (Diptera: Simuliidae). Universidad Distrital Francisco José de Caldas, Bogotá-Colombia

10. Rodríguez, C. T. (1999). Biodiversidad del relicto "Cedro Rosado" de la Universidad del Quindío, Biología y Educación (9):17-18.

11. Bueno M.L. (2002). Cromosomas Politénicos, En: Manual de guías de laboratorio de genética. Universidad Nacional de Colombia.

12. Moreno C. (1990) Estudio citogenético de Gigantodax ortizi Wygodzinsky, (Diptera: Simulidae) de la región de Chisacá. Trabajo de grado (Biólogo) Universidad Nacional de Colombia, facultad de Ciencias, Departamento de Biología..

13. Moreno C. (1999) Los cromosomas politénicos como herramienta para el estudio de especies de la familia Simuliidae. SOCOLEN (Sociedad Colombina de Entomología). XXVI, 137-146. 6-30-. Santafé de Bogotá..

14. Muñoz P. (1990). La importancia de los Cromosomas politénicos en la determinación taxonómica de los Simuliidos. Revista de la Academia Colombiana de Ciencias exactas, Físicas y Naturales 17(66), 511-522.

15. Henry W., Dey S., Varma R., Thapa S. y Procunier W. (2010) Polytene chromosomes of an 
Indian Himalayan black fly Simulium (Nevermannia) praelargum (Diptera: Simuliidae. Current Zoology 56 (4): 437-444.

16. Chaliow K, Unchulee L, y Visut B. (1966) Cytotaxonomy of Simulium siamense Takaoka and Suzuki (Diptera: Simuliidae) in Thailand. Genome 51: 972-987, 2008 Dunbar R W. Nature, 209, 597-599.

17. Mustapha M, Post RJ, y Krüger A. (2004). The cytotaxonomy and morphotaxonomy of Simulium mengense (Diptera: Simuliidae). Annals of Tropical Medicine \& Parasitology. 98(5) 509-523.

18. Levan A, Freda K y Sandberg A. (1964).Nomenclature for centromeric position of chromosomes. Hereditas (52) 201-220.

19. Romero-Zarco, C.( 1986) A new method for estimating karyotype asymmetry. Revista Taxon (35) 526-531. 1986.

20. Pelling C. (1969). Chromosomal synthesis of ribonucleic acid as shown by the incorporation of uridine labeled with tritium. Nature London (184): 655-656. 